Udams C.B.

I Catalogue of famaica Shells

11. do (iartier catulogue).

11.) Catalofie of Panama Shells. 
T. 3

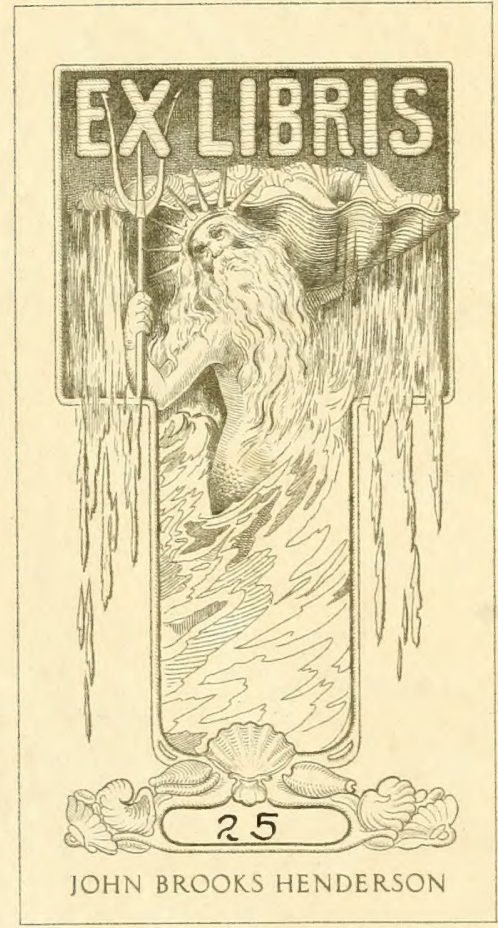



4uDiq!] [Dนouppos

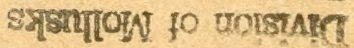




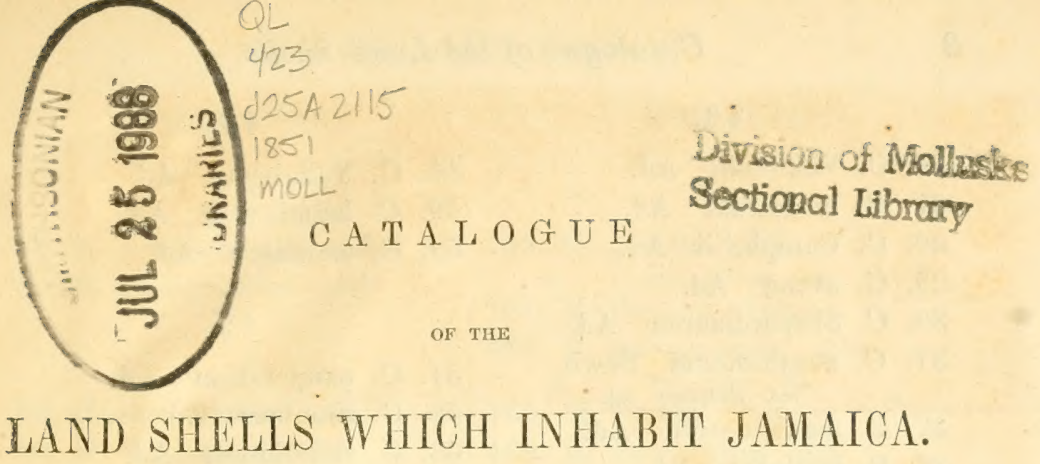

B Y C. B. A D A MS, PROFESSOR OF ZOOLOGY, ETC., IN AMHERST COLLEGE, MASSACHUSETTS.

APRIL,

\section{FAMILY CYCLOSTOMID平。}

\section{CYCLOSTOMA.}

$\$ 1$.
13. C. retrorsum Ad.

14. C. pisum Ad.

Syn. C. virgineum Ad.

1. C. anomalum Ad.

$\$ 2$.

2. C. pulchrum Wood.

3. C. fimbriatulum Sowb. a. var. docens Ad. b. var. albinodatum Ad.

4. C. Chittyi Ad.

5. C. amabile Ad.

6. C. Hillianum Ad. a. var. aculeosum Ad. b. var. leporilabre Ad.

7. C. lincinellum Lam. Syn. (?) C. spinulosum Ad.

8. C. Moussonianum Ad.

9. C. scabriculum Sowb. Syn. C. amandum Ad.

10. C. lincinum Linn.

11. C. lima Ad.

12. C. Blandianum Ad.

$\$ 3$.

15. C. variabile Ad. a. var. concentricum Ad.

16. C. intermedium Ad.

17. C. Grayanum Ad.

Syn. C. obscurum Gray.

18. C. serriferum Ad.

19. C. granosum Ad.

Syn. C. nodulosum Ad.

a. var. aberrans Ad.

20. C. pulchrius Ad.

Syn. C. Binneyanum Pfr.

21. C. mutatum Ad.

Syn. C. arliculatum Sowb.

22. C. mirabile Wood.

Syn. (?) C. decussatulum Ad.

23. C. mirandum Ad.

24. C. moribundum Ad.

25. C. monstrosum Ad. 


\section{$\$ 4$.}

26. C. Vilkinsoni Ad.

27. C. modestum Ad.

28. C. Campbellii Ad.

29. C. avena Ad.

30. C. Shepardianum Ad.

31. C. xanthostoma Sowb.

$$
\text { a. var. albilabre Ad. }
$$

32. C. tenuistriatum Ad.

33. C. ignilabre Ad.

$$
\text { a. var. albilabre Ad. }
$$

$$
\$ 5 \text {. }
$$

34. C. Newcombianum Ad.

35. C. fascia Gray.

a. var. proximum Ad.

36. C. Augustæ Ad.

$$
\begin{aligned}
& \text { a. var. nitens Ad. } \\
& \text { b. var. testudineum Ad. } \\
& \text { c. }
\end{aligned}
$$

37. C. sagittiferum Ad.

38. C. thysanoraphe Sowb.

39. C. Jayanum Ad.

$$
\text { Syn. C. solidum Ad. non Mke. 64. C. muticum Ad. }
$$

$\begin{array}{ll}a . & \text { var. rufilabre } \mathrm{Ad} \text {. } \\ \text { b. } & \text { var. nigrilabre Ad. }\end{array}$

40. C. Redfieldianum Ad.

41. C. labeo Múll.

42. C. Humphreysianum Pfr.

$$
\text { Syn. C. pictum Sowb. }
$$

43. C. interruptum Lam. non

$$
\text { Deless. }
$$

Syn. C. ambiguum Deless,

Chenu. non Lam.

44. C. Chevalieri Ad.

$$
\begin{aligned}
& a . \quad \text { var. album } A d . \\
& b \text { var. virgatum } A d .
\end{aligned}
$$

\section{\$ 6.}

45. C. album Lam.

$$
\text { a. var. fuscum Ad. }
$$

46. C. Bronnii Ad.

$$
\text { a. var. fuscolineatum Ad. }
$$

47. C. Bānksianum Sowb.

a. var, hyacinthinum $\boldsymbol{A d}$.
48. C. Yallahense Ad.

49. C. lamellosum Ad.

50. C. tectilabre Ad.

$$
8 .
$$

51. C. pauperatum Ad.

52. C. simulans Ad.

53. C. crenulosum $\mathrm{Ad}$.

54. C. columna Wood.

55. C. Sauliæ Sowb.

56. C. sericinum Ad.

57. C. maritimum Ad.

58. C. aurora Ad.

59. C. Tappanianum Ad.

60. C. Adamsi Pfr.

61. C. feeundum Ad. a. var. distinetum Ad.

62. C. Griffithianum Ad.

63. C. mordax Ad.

65. C. armatum Ad.

66. C. papyraceum Ad.

$$
\text { a. var. abbreviatum Ad. }
$$

$\$ 9$.

67. C. Duffianum Ad̀.

68. C. corrugatum Sowb.

69. C. jugosum Ad.

70. C. pallescens Ad.

71. C. subrugosum Sowb.

72. C. Jamaicense Chem.

73. C. seminudum Ad.

74. C. varians Ad.

75. C. crassum Ad.

76. C. perpallidum Ad.

7\%. C. asperulum Sowb.

78. C. dubiosum Ad.

79. C. suturale Sowb. 
STOASTOMA.

$\$ 1$.

S0. S. Agassizianum Ad.

81. S. Philippianum Ad.

\$2.

82. S. Gouldianum Ad.

83. S. Vilkinsonize Ad.

84. S. Tappanianum Ad.

85. S. Blandianum Ad.

S6. S. Hollandianum Ad.

87. S. Anthonianum Ad.

88. S. Fadyenianum Ad.

89. S. Petitianum Ad.

90. S. Pfeifferianum Ad. Dowd

91. S. Cumingianum Ad.

92. S. Chittyanum Ad.

93. S. pisum Ad.

94. S. Lindsleyanum Ad.

95. S. Redfieldianum Ad.

96. S. Moricandianum Ad.

97. S. Jayanum Ad.

98. S. Leanum Ad.

\section{LUCIDELLA.}

99. L. aureola Gray.

a. var. granulosa Ad.

\section{TROCHATELLA.}

100. T. Tankervillii Gray. 101. T. pulchella Gray.

a. var. multicarinata Ad.

b. var. nobilis Ad.

102. T. Grayana Pfr.

103. T. Josephinæ Ad.

a. var. pulchra Ad.

104. T. tenuis Ad.
HELICINA.

\& 1.

105. H. palliata Ad.

a. var. labiosa Ad.

106. H. Brownii Gray.

107. H. consanguinea Ad.

a. var. soror Ad.

108. H. hirsuta Ad.

109. H. microstoma Ad.

110. H. Hollandi Ad.

111. H. pusilla Ad.

112. H. macilenta Ad.

\section{$\$ 2$.}

113. H. maxima Sowb. a. var. eitrina Ad.

114. H. nobilis Ad.

115. H. albolabris Ad.

116. H citrinolabris Ad.

117. H. dubiosa Ad.

a. var. intermedia Ad.

118. H. solitaria Ad.

Syn. H. Guildingiana Pfs.

119. H. affinis Ad.

120. H. megastoma Ad.

121. H. Gossei Pfr.

$\$ 3$.

122. H. Adamsiana Pfr.

123. H. depressa Gray.

Syn. H. Leana Ad.

a. var. valida Ad-

124. H. lineata Ad.

5) 4 .

125. H. neritella Lam.

a. var. angulata Ad.

126. H. ampliata Ad.

127. H. Jamaicensis Sowb.

128. H. aurantia Gray.

$\$ 5$.

129. H. costata Sowb. 


\section{TRUNCATELLA.}

130. T. scalaris Mich.

Syn. T. Cumingii Ad.

Syn. T. costata Pfr.

131. T. modesta Ad.

132. T. Adamsi Pfr.

Syn. T. scalariformis Ad.

133. T. succinea Ad.

GEOMELANIA.

$\$ 1$.

134. G. Greyana Ad.

\section{§2.}

135. G. fortis Ad.

136. G. Jamaícensis Pfí

137. G. magna Ad.

138. G. procera Ad.

139. G. gracilis Ad.

a. var. parva Ad.

140. G. typica Ad.

a. var. pygma $\mathrm{Ad}$.
141. G. expansa Ad.

142. G. affinis Ad.

143. G. media Ad.

144. G. vicina Ad.

\$3.

145. G. minor Ad.

146. G. Hilliana Ad.

147. G. exilis Ad.

\$ 4 .

148. G. pauperata Ad.

149. G. Beardsleana Ad.

150. G. pygmæa Ad.

$\$ 5$.

151. G. comica Ad.

152. G. pyramidata Ad.

$\$ 6$.

153. G. costulosa Ad.

154. G. striosa Ad.

155. G. elegans Ad.

\section{FAMILY HELICIDZE.}

\section{CYLINDRELLA.}

$\$ 1$.

156. C. gracilis Wood.

157. C. elongata Chem.

Syn. C. Cumingíi Ad.

Syn. C. Chemnitziana (Helix) Fer.

$\$ 2$.

158. C. Agnesiana Ad.

a. var. diminuta Ad.

159. C. alba Ad.

a. var. stríatula Ad.

160. C. subula Fer.

[161. C. alabastrina Pfr.

162. C. Hurnboldtiana, var, $\beta$ Pfr.

163. C. variegata, var. $\gamma$ Pfr.]
$\$ 3$.

164. C. seminuda Ad.

165. C. Robertsi Ad.

166. C. costulata Ad.

167. C. costulosa Ad.

$\$ 4$.

168. C. Hydeana Ad. Syn. Bulimus Gossei Pfr.

$$
\$ 5 .
$$

169. C. Gossei Pfi.

170. C. Maugeri Wood.

$\begin{array}{ll}\text { a. } & \text { var. nigrescens Ad. } \\ \text { b. } & \text { var. solida Ad. } \\ \text { c. } & \text { var. levis Ad. } \\ \text { d. } & \text { var. tricolor Ad. }\end{array}$




$\begin{array}{ll}\text { e. } & \text { var. crassa Ad. } \\ \text { f. } & \text { var. unicincta Ad. } \\ \text { g. } & \text { var. bicincta Ad. } \\ h . & \text { var. concinna Ad. } \\ i . & \text { var. sinuata Ad. } \\ j . & \text { var. rufilabris Ad. } \\ k . & \text { var. citrina Ad. } \\ \ell . & \text { var. fusiformis Ad. } \\ m . & \text { var. albida Ad. } \\ n . & \text { var. valida Ad. } \\ \text { o. } & \text { var. unicolor Ad. } \\ p . & \text { var. striatula Ad. } \\ q . & \text { var. striata Ad. } \\ r . & \text { var. corpulenta Ad. } \\ s . & \text { var. fusca Ad. } \\ t . & \text { var. conica Ad. } \\ u . & \text { var. tesseiata Ad. } \\ v . & \text { var. cineren Ad. } \\ z . & \text { var. gracilis Ad. } \\ x . & \text { var. integra Ad. } \\ & \end{array}$

171. C. Hollandi Ad.

a. var. Augustæ Ad.

172. C. procera Ad.

173. C. montana Ad.

174. C. rosea Pfi.

a. var. magna Ad.
b. var. major Ad.
c. var. fortis Ad.
d. $\quad$ var. ambigua Ad.

175. C. cylindrus Chem.

176. C. rubella Ad.

177. C. zonata Ad.

178. C. aspera Ad.

179. C. brevis Pfr.

a. var. obesa Ad.
b. var. columna Ad.
c. $\quad$ var. intermedia $A d$.

180. C. Gravesii Ad.

181. C. lata Ad.

a. var. producta Ad.

182. C. nobilior Ad.

183. C. carnea Ad.

$$
\text { a. var. cerina Ad. }
$$

184. C. sanguinea Pfr.

185. C. pupæformis Ad.

$$
\$ 7 .
$$

186. C. humilis Ad.
187. C. inornata Ad.

188. C. simplex Ad. ?

$\$ 8$.

189. C. pusilla Ad.

190. C. rubra Ad.

191. C. elatior Ad.

192 C. princeps Ad.

193. C. Dunkeri Pfr.

194. C. similis Ad.

195. C. tenella Ad.

196. C. tenera Àd.

\section{ACHATINA.}

197. A. solitaria Ad.

198. A. ligata Ad.

199. A. Jamaicensis Pfr.

200. A. procera Ad.

201. A. Griffithii Ad.

202. A. Philippiana Pfr.

203. A. elegans Ad.

204. A. venusta Ad.

205. A. leucozonias Walch.

206. A. Dominicensis Gm.

207. A. nitida Ad.

208. A. angiostoma Ad.

209. A. Ingallsiana Ad.

210. A. unicolor Ad.

211. A. Gayana Ad.

212. A. nemorensis Ad.

213. A. similis $\mathrm{Ad}$.

214. A. Phillipsii Ad.

215. A. arcuata Pfr.

216. A. puella Ad.

217. A. tenera Ad.

218. A. longispira $\mathrm{Ad}$.

219. A. proxima Ad.

220. A. Blandiana Ad.

221. A. propinqua Ad. 
222. A. osculans Ad. $\S 2$.

223. A. perplexa Ad. 224. A. Gossei Pfr.

225. A. costulata Ad. 226. A. gracilior Ad.

227. A. costulosa Ad. 225. A. curvilabris Pfr.

229. A. vicina Ad:

230. A. peliucens Ad.

231. A. levis Ad.

232. A. micans Ad.

233. A. iota Ad.

234. A. octona Chem.

235. A striosa Ad.

\section{SPIRAXIS.}

236. S. costulosa Ad.

237. S. inusitata (Achatina) Ad.

238. S. mirabilis (Bulimus) Ad.

239. S. anomala (Achatina) Ad.

240. S. brevis Ad.

241. S. aberrans (Achatina) Pfr.

a. var. unicolor Ad.

\section{BULIMUS.}

$\S 1$.

242. B. minimus Ad.

243. B. striatellus $\Lambda d$.

244. B. terebella Ad.

245. B. pauperculus Ad.

246. B. hortensis Ad.

247. B. octonoides Ad.

248. B. pallidus Ad.

249. B. monodon Ad.

250. B. nitidiusculus Ad.

251. B. subula Pfr.

Syn. B. procerus Ad.

252. B. læviusculus Ad.
253. B. rufescens Gray.

254. B, erubescens Pfr.

255. B. immaculatus Ad.

256. B. zebra Müll.

syn. B. undatus Brug.

\$ 3.

25\%. B. macrospira Ad.

PUPA.

$\$ 1$.

258. P. exilis Ad.

$\$ 2$.

259. P. fallax (?) Say.

260. P. lata Ad.

261. P. contracta (?) Say.

262. P. tenuidens Ad.

263. P. hexodon Ad.

264. P. servilis Gould.

265. P. Jamaicensis Ad.

$\$ 3$.

266. P. striatella (?) Pfr.

\section{SUCCINEA.}

267. S. angustior Ad.

268. S. Sagra (?) Orb.

269. S. latior Ad.

270. S. contorta Ad.

HELIX.

$\$ 1$.

271. H. Martiniana Pfr.

272. H. peracutissima Ad.

273. H. soror Fer.

274. H. cara Ad.

a. var. media Ad.
b. var. amabilis Ad.


275. H. Chittyana Ad.

276. H. patina Ad.

var. nobilis $A \mathrm{~d}$.

277. H. fluctuata Ad.

?78. H. ingens Ad.

r. var. imperforata Ad.

b. var. indigna Ad.

279. II. acuta Lam.

a. var. acutissima Lam.

2S0. H. fuscolabris Ad.

2\&1. II. lucerna Müll.

282. H. fortis $\mathrm{Ad}$.

283. H. Okeniana Pfr.

284. H. Swainsoniana Ad.

285. H. tridentina Fer

286. H. Bronni Pfr.

227. H. valida Ad.

285. H. consanguinea Ad.

a. var. imperfurala Ad.

b. var. carinata Ad.

289. II. picturatie Ad.

290. H. invalida Ad.

291. H. sinuata Müll.

a. rar propenuda Ad.

b. var. candescens Ad.

292. H. strangulata Ad.

293. II. anomala Pfr.

a. var. pallescens $\mathrm{dd}$.

b. var. convexa Ad.

294. H. Bainbridgei Pfr.

a. var. pretiosa Ad.

295. H. Spengleriana Pfr.

$\$ 2$.

296. H. Redfieldiana Ad.

$\$ 3$.

297. H. aspera Fer.

\section{$\$ 4$.}

298. H. Jamaicensis Chem. syn. H. pulla Gm.

$\$ 5$.

299. H. angustata Fer.
300. H. Cubensis, var. o Pr.

301. H. tunicata Ad.

302. H. Buddiana Ad.

303. H. MacMurrayi Ad.

304. H. munda Ad.

305. H. columellata Ad.

306. H. tenerrima Ad.

307. H. graminicola $\mathrm{Ad}$.

308. H. nemoraloides Ad.

309. H. pulchrior Ad.

310. II. Gossei Pfr.

311. H. Blandiana : Ad.

312. H. subconica Ad.

313. H. fuscocineta Ad.

314. H. virginea Ad.

315. H. Jacobensis Ad.

316. H. fuscula Ad.

$\S 6$.

317. H. immunda Ad.

318. H. rufula Pfr.

319. H. ptychodes Pfr.

320. H. alveus Ad.

$\$ 8$.

321. H. perdepressa Ad.

322. H. brevior Ad.

Syn. II. depressa Ad.

323 . H. sincera Ad.

324. H. diminuta Ad.

325. H. apex Ad.

326. H. inconspicua Ad.

327. H. peraffinis Ad.

328. H. Boothiana Pfr.

Syn. H. pellucida Ad.

329. H. spreta Ad.

$$
\text { a. var. errans. }
$$

330. H. turbiniformis Pfr.

Syn. H. subpyramidalis Aci

331. H. Anthoniana Ad. 
332. H. angustispira Ad.

333. H. brevis Ad.

33. H. Hollandi Ad.

335. H. similis Ad.

336. H, arboreoides Ad.

337. H. ambigua Ad.

338. H. osculans Ad.

a. vir. delaminata Ad.

$\$ 9$.

339. H. lamellifera Ad.

310. H. pila Ad.

341. H. Foremaniana Ad.

312. H. Cookiana Gm.

343. H. epistylioides Fer.

314. H. alligans Ad.

$3+5 \mathrm{H}$. epistylium Müll.

a. var. minor Ad.

b. var. delaminata $\mathrm{Ad}$.
346. H. torrefacta Ad.

347. H. comnectens Ad.

348. H. epistyliulum Ad.

$\$ 10$.

349. H. simulans Ad.

350. H. dioscoricola Ad.

\$) 11 .

351. II. paludosa Pfi.

PROSERPINA.

352. P. discoidea Ad.

353. P. opalina (Helix) Ad.

304. P. nitida Sowb.

a. var: planulata Ad.

355. P. pulchra Ad.

356. P. bidentata Ad.

357. P. pisum Ad.

\section{FAMIIY AURICUIIDF。}

\section{MELAMPUS.}

358. NI. coniformis Brug.

359. MI. flavus $\mathrm{Gm}$.

Syn. Auricula monile Lam. 360. AI. pusillus Gm.
361: MI. coronatus Ad. 362. M. cingulatus Pfr.

PEDIPES.

363. P. quadridens Pfr. 364. P. ovalis Ad. 


\section{CATALOGUE OF THE FRESII WATER SHELLS WHICH INHABIT JAMAICA.}

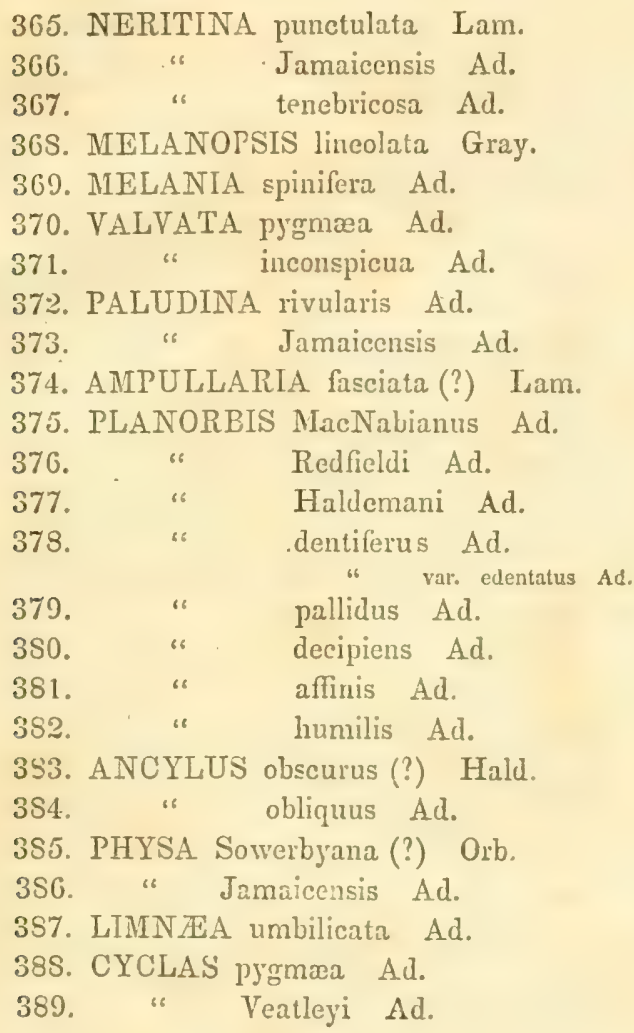

Norice.-The Hon. Edward Chitty of Jamaica havines furnished the writer with his duplicates of the land and fresh-water shelts of Jamaica, so far as necessary for makine out suites, a few collections, each containing nenrly all the discovered species, - abut 500 species and varieties,will be put up for sale, at prices from $\$ 100$ to $\$ 1.50$. Sultes of single genere, and miscellaneous pnreels for setection, (with the priviluge of returning stuch shello as may not be wanted,) can also be furnished. It is proper to adt, that the olject of the sales is not pecuniary profit, but to theet the expenses of an illustrated monograph, the outlay for which probably will much exceed the receipts. 



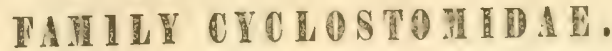

\section{CYCLOSTOMA.}

1. C. pulchrum Wood.

2. C. fimbriatulum Sowb.

3. C. Chittyi Ad.

4. C. aculeosum $\mathrm{Ad}$.

5. C. spinulosum Ad.

6. C. amandum Ad.

7. C. Hillianum Ad.

8. C. amabile $\mathrm{Ad}$.

9. C. scabriculum Somb.*

10. C. lincinum Limn.

11. C. $\operatorname{lima} \mathrm{Ad}$.

12. C. Blandianum Ad.

13. C. lincinellum Lam.*

14. C. variabile Ad.

15. C. concentricum Ad.

16. C. Grayanum Ad.

17. C. serriferum $A d$.

18. C. pulchrius Ad.

19. C. articulatum Sowb.

20. C. decussatulum $\Lambda d$.

21. C. mirandum $A d$.

22. C. moribundum Ad.

23. C. intermedium Ad.

24. C. monstrosum Ad.

25. C. Vilkinsonii Ad.

26. C. avena Ad.

27. C. modestum Ad.

28. C. ambiguum Lam(?)Chenu.

29. C. thysanoraphe Sowb.

30. C. pictum Humph.

31. C. Jayanum $\dagger$ Ad.

32. C. labeo Lam.*

33. C. Augustæ Ad.

34. C. fascia Gray.

35. C. proximum Ad.

36. C. Neweombianum Ad.

37. C. nitens Ad.

38. C. xanthostoma Sowb.

39. C. tenuistriatum Ad.

40. C. ignilabre Ad.

41. C. pisum Ad.
42. C. Bronnii Ad.

43. C. fuscolineatum Ad.

44. C. album Lam.

\{45. C. hyacinthinum Ad.

$\{$ 46. C. Banksianum Sowb.*

47. C. Redfieldianum Ad.

48. C. tectilabre Ad.

49. C. pauperatum Ad.

50. C. crenulosum Ad.

51. C. quinquefasciatum Ad。

52. C. Saulix Sorvb.*

53. C. mordax Ad.

54. C. maritimum Ad.

55. C. aurora Ad.

56. C. fecundum Ad.

57. C. Adamsi Pfr.

58. C. muticum Ad.

59. C. armatum Ad.

60. C. Dufianum $\Lambda$ d.

61. C. corrugatum Sorrb.

62. C. Jamaicense Sowb.

63. C. asperulum Sorvb.*

\section{HELICINA.}

64. H. palliata Ad.

65. H. Brownii Gray.

66. H. Hollandi Ad.

67. H. macilenta $\mathrm{Ad}$.

68. H. maxima Sorvb.

69. H. albolabris $\mathrm{Ad}$.

70. H. citrino-labris $\mathrm{Ad}$.

71. H. solitaria Ad.

72. H. affinis $\mathrm{Ad}$.

73. H. megastoma $\mathrm{Ad}$.

74. H. Gossei Pfr.

75. II. Adamsiana Pfr.

76. I. depressa Gray.

77. H. lineata Ad.

78. H. neritella Lam.

79. II. aurantia Gray.

80. H. Jamaicenais Sntrh. 
89. S. Blandianum Ad.

81. H. costata Sowb.

'TROCHATELLA.

82. T. Tankervillii Gray.

83. T. pulchella Gray.

84. T. Gravana Pfr.*

85. T. Josephinæ Ad.

86. T. tenuis Ad.

\section{LUCIDELLA.}

87. I. aureola Gray.

STOASTOMA.

88. S. Gouldianum Ad.

90. S. Fadyenianum Ad.

91. S. Pfeifferianum Ad.

92. S. Cumingianum Ad.

93. S. Chittyanum Ad.

94. S. pisum Ad.

95. S. Lindsleyanum Ad,

96. S. Redfieldianum Ad.

97. S. Jayanum Ad.

98. S. Leanum Ad.

\section{TRUNCATELLA.}

99. T. scalaris Mich.

100. T. Adamsi Pfr.

101. T. succinea Ad.

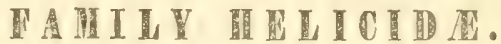

\section{GEOMELANIA.}

102. G. Jamaicensis Pfi。

103. G. minor Ad.

104. G. expansa Ad.

105. G. elegans Ad.

\section{CYLINDREILA.}

106. C. pygmra Ad.

107. C. Beardsleana Ad。

108. C. gracilis Wood.

109. C. elongata Chem.

110. C. Agnesiana Ad.

111. C. alba $\mathrm{Ad}$,

112. C. Gossei Pfr.

113. C. seminuda Ad.

114. C. costulata Ad.

115. C. subula Fer.**

116. C. alabastrina $\mathrm{Pfr}$ **

117. C. Humboldtiana, var. $\beta$ Pfr.* 142. A. Jamaicensis Pfr.

118. C. variegata, var. $\gamma$ Pfr.* 143. A. procera Ad.

119. C. montana Ad. 144. A. Griffithii Ad.

120. C. Gravesii Ad. 145. A. Phillipiana Pfr。

121. C. rosea Pfr.

a." " var. magna Ad. 148. A. leucozonias Walch.

b. " " "major Act. 11!). A. Dominicensis Gm.

122. C. ambigua Ad.

123. C. cylindrus Chem.

124. C. rubella Ad.

125. C. aspera Ad.

126. C. obesa Ad.

127. C. brevis Pfr.
146. A. elegans Ad.

147. A. venusta Pfr.

128. C. columna Ad.

129. C. nobilior Ad.

a. " " var.cerina $\mathrm{Ad}$

131. C. sanguinea Pfr.

132. C. Maugeri Wood.

133. C. inornata Ad.

134. C. simplex Ad.

135. Hydeana Ad.

136. C. rubra Ad.

137. C. Dunkeri Pfi。

138. C. similis Ad.

139. C. tenella Ad.

140. C. tenera Ad.

\section{ACHATINA.}

150. A. curvilabris $\mathrm{Pfr}$.

151. A. angiostoma Ad.

152. A. Ingalisiana Ad.

153. A. unicolor Ad.

144. A. nemorensis Ad,

155. A. Phillipsii Ad. 
156. A. arcuata Pfr.

157. A. vicina Ad.

158. A. propinqua Ad.

159. A. Gossei Pfr.

160. A. costulata Ad.

161. A. aberrans Pfr.

162. A. pellucens Ad.

163. A. levis Ad.

164. A. micans. Ad.

165. A. iota Ad.

166. A. octona Chem.

167. A. striosa. Ad.

168. A. inusitata Ad.

\section{BULIMUS.}

169. B. minimus $A d$.

170. B. striatellus Ad.

171. B. terebella Ad.

172. B. pauperculus Ad.

173. B. octonoides Ad.

174. B. pallidus Ad.

175. B. monodon Ad.

176. B. nitidiusculus Ad.

177. B. subula Pfr.

178. B. Irviusculus Ad.

179. B. rufescens Gray.**

180. B. erubescens Pfr.

181. B. immaculatus Ad.

182. B. zebra Mull.

183. B. anomalus Ad.

184. B. mirabilis Ad.

\section{PUPA.}

185. P. fallax (?) Say.

186. P. lata Ad.

187. P. contracta (?) Say.

188. P. tenuidens Ad.

189. P. hexodon Ad.

190. P. servilis Gould

191. P. Jamaicensis Ad.

192. P. exilis Ad.

193. P. striatella (?) Fer.

\section{SUCCINEA.}

194. S. angustior Ad. 195. S. Sagra Orb.
196. S. latior Ad.

197. S. contorta Ad.

\section{HELIX.}

198. H. Martiniana Pfr.*

199. H. peracutissima Ad.

200. H. soror Fer.

201. H. cara Ad.

292. H. Chittyana Ad.

203. H. patina Ad.

204. H. fluctuata Ad.

205. H. acutissima Lam.

206. H. Bainbridgei Pfr.

207. H. Spengleri Hinds."

208. H. lucerna Mull.

209. H. Okeniana Pfr.*

210. H. tridentina Fer.

211. II. Bronnii Pfr.

212. H. picturata Ad.

213. H. sinuata Mull.

214. H. strangulata Ad.

215. H. anomala $\mathrm{Pfr}$.

216. H. aspera Fer.

217. H. Jamaicensis Chem.

218. H. angustata Fer.**

219. H. Cubensis, var. $\vartheta$ Pfr.*

220. H. tunicata Ad.

221. H. sulphurea Ad.

222. H. MacMurrayi Ad.

223. H. munda Ad.

224. H. tenerrima Ad.

225. H. graminicola Ad.

226. H. nemoraloides Ad.

227. H. Gossei Pfr.

228. H. subconica Ad.

229. H. fuscocincta Ad.

230. H. virginea Ad.

231. H. fuscula Ad.

232. H. immunda Ad.

233. H. ptychodes Pfr.

234. H. perdepressa Ad.

235. H. brevior Ad.ई

236. H. sincera Ad.

237. H. diminuta Ad.

238. H. apex Ad.

239. H. inconspicua $\mathrm{Ad}$. 
240. H. peraffinis $A d$.

241. H. Bonthiana Pfr.

142. II. turbiniformis Pfr.

243. II. Anthoniana Ad.

244. II. angustispira Ad.

245. II. ambigua $\mathrm{Ad}$.

246. II. brevis Ad.

247. II. Hollandi Ad.

218. II. arboreoides Ad.

249. II. similis Ad.

250. II. lamellifera Ad.
251. H. pila Ad.

252. H. Cookiana Gm.

253. H. torrefacta Ad.

254. H. epistyliulum Ad.

255. H. epistylium Mull.

256. H. epistylioides Fer.

257. H. simulans Ad.

258. H. dioscoricola Ad.

\section{PROSERPINA.}

259. P. opalina Ad. 260. P. nitida Sowb.

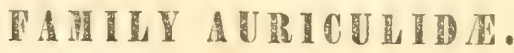

\section{MELAMPUS.}

261. M. coniformis Brug.

262. M. flavus $\mathrm{Gm}$.

263. M. pusillus Gm.

264. M. coronatus Ad.
265. M. cingulatus Pfr.

\section{PEDIPES.}

266. P. quadridens Pfr.

267. P. ovalis Ad.

* Not in the Zoological Museum of Amherst College.

$\dagger$ Syn. C. solidum Ad.

$\S$ Syn. H. depressa Ad.

CATALOGUE OF FRESII WATER SHELLS, WHICH INHABIT J A A I CA.

1. NERITINA punctulata Lam.

2. MELANOPSIS lineolata Gray.

3. MELANIA spinifera Ad.

4. VALVATA pygmæa Ad.

5. PALUDINA rivularis Ad.

6. " Jamaicensis Ad.

7. AMPULLARIA fasciata (?) Lam.

8. PLANORBIS MacNabianus Ad.

9. " " Redfieldi Ad.

10. " Haldemani Ad.

11. " dentiferus Ad.

12. " pallidus Ad.

13. " decipiens Ad.

14. " affinis Ad.

15. ANCYLUS obscurus (?) Hald.

16. PHYSA Soverbyana Orb.

17. LIMNAEA umbilicata Ad.

18. CYCLAS pygmia $A d$.

19. " Veatleyi Ad.

[F This Catalogue is reprinted in this form for convenience in exchanges. Please return one copy with $a$ * prefixed to the numbers of your desidernta. 


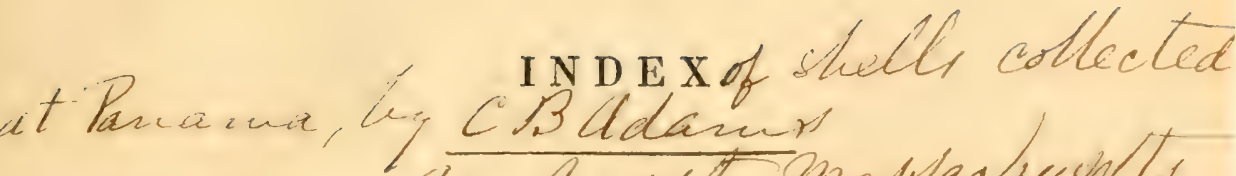

Family Cypraeidie.

No.

1. O. avena Sowb.,

2. emarginata Sowb.,

3. neglecta Ad.,

4. variabilis Ad.,

5. sp. indet.,

OYPREA.

6. C. arabicula Lam.,

7. cervinetta Kr.,

8. punctulata Gray,

9. pustulata Lam.,

10. radians Lam.,

11. rubescens Gray,

12. sanguinea Gray,

ERATO.

13. E. cypræola Sowb.,

MARGINELLA.

14. M. minor Ad.,

15. sapotilla Hinds,

\section{Family Mitridre.}

MITRA.

16. M. funiculata $R \nabla$,

17. lens Wood,

18. nucleola Lam.,

19. solitaria $\mathrm{Ad}_{\text {, }}$

20. tristis Brod,

Family Purpuridae.

TEREBRA.

21. T. elata Hinds,

22. lartæformis Hinds,

23. robusta Hinds,

24. speeillata Hinds,

25. tuberculosa Hinds,
No.

26. T. varicosa Hinds,

27. sp. indet, $a$,

28. " $b$,

29. " $c$

30. " d,

$31 . \quad " e_{2}$

OLIVA.

32. $O$, angulata Lam.,

33. araneosa Lam.

34. inconspicua Ad.,

35. pellucida $R \nabla$.

36. porphyria Linn.,

37. semistriata Gray,

38. testacea Lam.,

39. undatella Lam.,

40. venulata Lan.,

41. volutella Lam.,

PLANAXIS.

42. P. planicostata Sowb.,

NASSA.

43. N. canescens Ad.,

44. collaria Gould,

$44 a$. " var. $a$,

45. corpulenta Ad.,

Page

47

47

47

47

47

47

46. gemmulosa Ad., $\quad 61$

47. glauca Ad., 61

48. luteostoma Brod., 62

49. nodifera Powis, 63

50. pagodus (Triton) Rv., $\quad 63$

51. Panamensis Ad., 64

52. proxima Ad., 64

53. scabriuscula (?) Powris, 65

54. striata Ad., 65

55. versicolor Ad., 66

55 a. " var, striatula Ad, 66

56. Wilsoni Ad.; 67

BUCCINUN.

57. B. crassum (Phos) Hinds, 67 
No.

58. B. distortum Wood,

59. insigne $\mathrm{Rr}$.,

60. lngubre Ad.,

61. pagodus $R v$.,

62. pristis Desh.,

63. ringens $R_{V}$.,

64. sanguinolentum Ducl.,

65. Stimpsonianum Ad,

DOLIUM.

66. D. ringens Swains,

MONOCEROS.

67. M. brevidentatum Wood,

68. cingulatum Wood,

PURPURA.

69. P. Carolensis Rv.,

70. foveolata Ada.,

71. kiosquiformis Ducl.,

72. sp. indet.,

73. melo Ducl.,

74. osculans Ad.,

75. tecta Wood,

76. undata Lam.,

\section{COLUMBELLA.}

77. C. atramentaria Sowb.,

78. bicanalifera Sowb.,

79. Boivinii $\mathrm{Kr}$., 。

80. conspicua Ad.,

81. costellata Sowb.,

82. diminuta Ad.,

83. dorsata Sowb.,

84. fluctuata Sowb.,

85. fulva Sowb.,

86. fuscata Sowb.

87. gibberula Sowb.,

88. gracilis Ad.,

89. guttata Sowb., posted C. cribraria Sowb.

90. hæmastoma Sowb.,

91. harpiformis Sowb.,

92. labiosa Sowb.,

93. lyrata Sowb.,

94. major Sowb.,

95. modesta Powis,

96. mosta Ad.,

97. nigricans Sowb.,

98. parva Sowb.,

\begin{tabular}{|c|c|c|}
\hline Page & No. & Page \\
\hline 68 & 99. C. pulchrior Ad., & 96 \\
\hline 69 & 100. pygmæa Sowb., & 97 \\
\hline 69 & rugosa Sowb., & 97 \\
\hline 70 & strombiformis Lam., & 98 \\
\hline 70 & tesselata Ad., & 99 \\
\hline 71 & 104. turrita Sowb., & 99 \\
\hline 72 & 105. varia Sowb., & 100 \\
\hline 72 & 106. sp. indet., & 101 \\
\hline 78 & \multicolumn{2}{|l|}{ RICINULA. } \\
\hline 18 & \multirow{2}{*}{$\begin{array}{l}\text { 107. R. carbonaria (?) Rv., } \\
\text { 108. jugosa Ad., } \\
\text { 109. Reeviana Ad., }\end{array}$} & $\begin{array}{l}101 \\
101\end{array}$ \\
\hline 74 & & 102 \\
\hline 75 & \multicolumn{2}{|l|}{ CASSIS. } \\
\hline & \multirow{2}{*}{$\begin{array}{l}\text { 110. C. abbreviata Lam., } \\
\text { 111. coaretata Sowb., }\end{array}$} & 102 \\
\hline 76 & & \\
\hline 77 & & \\
\hline
\end{tabular}

112. O. tubereulosa Sowb.,

104

\section{Family Strombidae.}

\author{
CONUS.
}

113. C. brunneus Wood, 104

114. gladiator Brod., 105

115. mahogani $R \nabla$., $\quad 105$

116. nux Brod., 106

117. princeps Linn., 106

118. purpurascens Brod., 108

119. regalitatis Sowb., $\quad 109$

120. regularis Sowb., $\quad 110$

121. vittatus Brug., $\quad 110$

STROMBUS.

122. S. galea Wood,

111

112

124. granulatus Wood, 113

125. Peruvianus Swains., 114

\section{Family IIuricidae.}

TRITON.

126. T. Chemnitzii Gray,

128. fusoides $\mathrm{Ad}$., 116

129. gibbosus Brod., 116

130. lignarius Brod., 117

131. vestitus Hinds, 118 
No.

132. R. cælata Brod.,

133. nana Brod.,

134. nitida Brod.,

135. plicata $R \nabla$. ,

MUREX.

136. M. dubius Sowb.,

137. erosus Brod,,

138. radix Gm.,

139. rectirostris Sowb.,

140. recurvirostris Brod.,

141. regius IVood,

142. salebrosus King,

143. vibex Brod.,

144. vittatus Brod,

PYRULA.

145. P. patula Brod,

FICULA.

146. F. ventricosa Sowb.,

FUsus.

147. F. bellus Ad.,

FASCIOLARIA.

148. F. granosa Brod.,

TURBINELLA.

149. T. crestus Brod,

150. castanea Gray,

151. cerata Wood,

152. rudis $R \nabla$. .,

153. spadicea $R \nabla$.,

CANCELLARIA.

154. C. affinis Ad.,

155. clavatula Sowb.,

156. decussata Sowb.,

157. goriostoma Sowb.,

158. mitriformis Sowb.,

159. pulchra Sowb.,

160. pygma $\mathrm{Ad}$,

161. solida Sowb.,

162. tesselata Sowb.,

PLEUROTOMA.

163. P. aterrima Somb.,

164. atrior Ad.,

165. bicanalifera Sowb.,

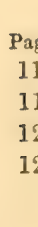

121

121

122

123

124

124

125

126

127

127

128

129

129

130

181

131

132

132

132

133

134

134

135

135

136

137

137

138

138

139
No.

166. P. collaris Sowb.,

167. concinna Ad.,

168. corrugata Sowb.,

169. diseors Sowb.,

170. duplicata Sowb.,

171. excentrica Sowb.,

172. exigua Ad.,

173. gemmulosa Ad.,

174. grandimaculata Ad.,

175. incrassata Sowb.,

176. nigerrima Sowb.,

177. obeliscus $R \nabla$.,

178. olivacea Sowb.,

179. pallida Sowb.,

180. rigida Hinds,

181. rudis Sowb.,

182. rustica Sowb.,

183. striosa Ad.,

184. zonulata $R \nabla$.,

185. sp. indet. $a$,

$186 . \quad$ " $b$,

\section{MANGELIA.}

187. M.sp. indet. $c$,

188. " $d$ "

189. " $\quad e$,

Pago

139

140

140

141

141

142

142

143

143

144

144

145

145

146

146

146

147

147

148

148

148

190. " $f$,

191. neglecta Ad.,

192. sulcosa? (Columbella) Sowb., 149

\section{CERITHUM.}

193. C. adustum $\mathrm{K} r$,

194. assimilatum Ad.,

195. bimarginatum Ad., 151

196. famelicum Ad., 152

197. gemmatum Hinds, 152

198. interruptum (?) Mke, 153

199. sp. indet., 153

200. irroratum Gould, 154

201. neglectum Ad., 154

202. Pacificum Sowb., 155

203. pauperculum Ad., 155

204. pulchrum Ad, 156

205. Reevianum Ad., 156

206. validum Ad., 157

TRIPIIORIS.

207. T. alternatus Ad.,

208. inconspicuus $\Lambda d$, 
No.

Family Littorinida.

210. T. Banksii $R$.,

CACUM,

211. C. diminutum $A d$,

212. eburneum Ad.,

213. firmatum $\mathrm{Ad}$.,

214. lave Ad.,

215. laqueatum Ad,

216. monstrosum Ad,

217. parvum Ad,

218. pygmæum Ad.,

CHEMNITZIA.

219 C. aculeus $\mathrm{Ad}$,

220. acuminata Ad.,

221. affinis $\mathrm{Ad}$,

222. clathratula Ad.,

223. communis Ad,

224. gracilior Ad.,

225. major Ad.,

226. marginata Ad.,

227. Panamensis Ad,

228. similis $\mathrm{Ad}$,

229. striosa $\mathrm{Ad}$.,

230. turrita Ad.,

LITTORINA.

231. L. angiostoma Ad.,

232. aspera Phil,

233. atrata Ad.,

234. conspersa Phil.,

235. excavata Ad.,

236. fasciata Gray,

237. foveata $\mathrm{Ad}$,

238. megasoma Ad.,

239. parrula (?) Phil,

240. pulchra Swains,

241. puncticulata Phil,

242. varia Sowb.,

RISSOA.

243. R. clandestina Ad,

244. firmata $\mathrm{Ad}$.,

245. fortis Ad.,

246. inconspicua $\mathrm{Ad}$,

247. infrequens Ad.,

248. Janus Ad,

\begin{tabular}{r|} 
Page \\
160
\end{tabular}

161

161

161

162

162

162

163

163

164

164

165

165

166

167

167

167

168

168

169

169

170

170

171

172

172

173

173

174

174

175

176

176

179
249. R. notabilis Ad.

Page

180

$\begin{array}{lll}\text { 250. scalariformis Ad., } & 180 \\ \text { 251. sp. indet., } & 181\end{array}$

\section{Family 'Trochidae.}

CINGULA.

252. C. inconspicua Ad.,

253. paupercula Ad.,

254. terebellum Ad.,

255. turrita Ad.,

LITIOPA?

256. I. saxicola Ad,

ADEORBIS.

257. A. abjecta Ad.,

VITRINELLA.

258. V. concinna Ad.,

181

181

182

182

259. exigua Ad.,

260. Janus Ad.,

261. minuta Ad.,

262. modesta Ad.,

263. Panamensis Ad.,

264. parva Ad.,

265. perparva Ad.,

266. regularis $\mathrm{Ad}$,

267. seminuda Ad.,

268. tricarinata $\mathrm{Ad}$,

269. valvatoides $\mathrm{Ad}$.,

SOLARIUM.

270. S. sp. indet. $\alpha$,

190

271. “ $\quad b, \quad 190$

$\begin{array}{llll}272 . & c, & 190\end{array}$

Trocuius.

273. T. catenulatus Phil., $\quad 190$

274. coronulatus Ad, 191

275. Leanus Ad., 191

276. lima Phil,, 192

277. lividus Phil., 192

278. Panamensis Phil, 193

279. pellis-serpentis Wood, 193

280. reticulatus Wood, 194

TURBO.

281. T. Buschii Phil., 195

282. phasianella Ad, 195

283. rutilus Ad., 196 
No.

284. T. sarosus Wood,

SCALARIA.

285. S. hexagona Sowb.,

286. obtusa Sowb.,

287. sp. indet. $a$,

288. " " $b$,

$289 . \quad$ " $c$,

Family Eulinidae.

EULINA.

290. E iota Ad.,

198

291. recta $\mathrm{Ad}$.,

292. solitaria Ad.,

\section{Family Tornatellidae.}

PYRAMIDELLA.

293. P. sp. indęt.,

294. conica Ad,

\section{Family Neritidae.}

NATICA,

295. N. Chemnitzii Mke.,

296. lurida Phil.,

297. otis Brod.,

298. Salangonensis (?) Recl.,

299. Souleyetiana Recl,

300. virginea? Recl.,

301. sp. indet. $a$,

$302 . \quad$ " $b$,

303. " $c$,

NERITA.

304. N. scabricosta Lam.,

305. sp. indet.,

NERITTNA.

306. N. Guayaquilensis Sowb.,

307. picta Sowb.,

\section{Family Amriculidae.}

PEDIPES,

308. P. angulata Ad,

AURICULA.

309. A. acuta Orb.,

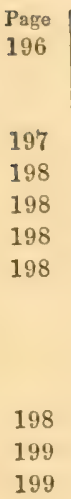

No.

310. A. concinna Ad,

311. infrequens Ad.,

312. Panamensis Ad.,

313. stagnalis Pet.,

314. Tabogensis Ad,

Page

208

209

209

210

211

212

315. trilineata Ad.,

316. sp. indet,

212

\section{Family Cyclostomidae.}

TRUNCATELLA.

317. T. Bairdiana Ad.,

318. dubiosa Ad.,

\section{Family Bullidae.}

BULLA.

319. B. infrequens $A d$.,

320. luticola Ad,

214

215

321. punctulata A. Ad,

215

322. sp. indet.,

215

\section{Family Vermetidae.}

323. V.glomeratus Rous.,

324. Panamensis Rous.,

216

216

\section{Family FHaliotidae.}

STOMATELLA.

325. S. inflata Ad.,

216

\section{Family Calyprraeida.}

HIPPONYX.

326. H. sp. indet.,

327. barbata Sowb.,

328. Panamensis Ad.,

329. radiata Sowb,

\section{CALYPTREA.}

330. C. aberrans Ad,

331. aspersa Ad,

332. cepacea Brod.,

333. conica Brod.,

334. dentata Mke,

335. hispida Brod.,

336. imbricata Brod.,

337. maculata Brod,

338. planulata Ad, 
No.

339. C. radiata Brod.,

340. regularis Ad,

341. umbrella Desh,

342. unguis (?) Brod,

CREPIDULA.

343. C. cerithicola Ad,

344. echinus Brod,

345. excavata Brod.,

346. hepatica (?) Desh.,

347. incurva Brod.,

348. Lessoni Brod,

349. squama Brod.,

350. unguiformis (?) Lam.,

351. nivea Ad,

352. osculans Ad.,

353. rostrata Ad.,

\section{Family Fissurellidae.}

FISSURELLA.

354. F. xqualis Sowb.,

355. alta Ad.,

356. macrotrema Sowb.,

357. mierotrema Sowb,

358. mus Rv.,

359. nigropunctata Sowb.,

360. ostrina Rv.,

361. virescens Sowb.,

\section{SIPIONARIA.}

362. S. characteristica Rv.

363. costata Sowb.,

364. gigas Sowb.,

365. maura Sowb.,

366. pica Sowb.,

LOTTIA.

367. L. patina (?) Rathke,

$367 a$ " var. $a$,

368. sp. indet. $a$,

369 . " " $b$,

$370 . \quad$ " $c$,

\section{Family Patellidae.}

371. P. sp. indet,

CHITON.

372. C. clathratus RV.,

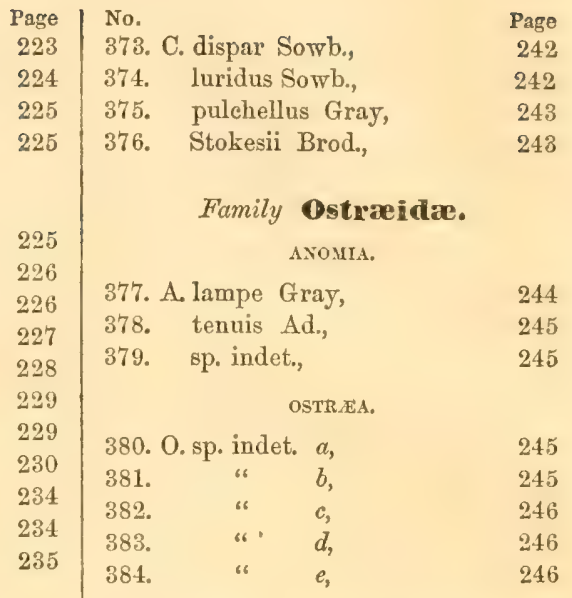

\section{Family Peetenidae.}

SPONDYLUS.

235

236

236

237

237

237

238

238

239

239

239

240

240

241

241

241

241

241

95. P. maura Sowb.

396. tuberoulosa Sowb.,

MXTILUS.

242 397. M. sp. indet,

AVICULA.

391. A. margaritifera?

PERNA.

393. P. sp. indet. $a$,

PINNA.
247

247

247

248

248

249

Family Aviculidae.

249

250

250

250

\section{Family Irytilidae.}

251 
LTTHODOMUs,

No.

398. L. sp. indet. $a$,

MODIOLA,

399. MI. semifusca (?) Lam.,

400. sp. indet. $a$,

401. " b)

402. " c,

403. "6 $d$,

404. " $e$,

\section{Family Clnamidre.}

CHAMA.

405. C. Buddiana Ad.,

406. corrugata Brod.,

407. echinata Brod.,

\section{Family Arcidae.}

NUCULA.

408. N. Elenensis Sowb.,

409. exigua Sowb.,

410. polita Sowb.,

PECTUNCULUS.

411. P. assimilis Sowb.,

412. maculatus (?) Brod.,

ARCA.

413. A. alternata Sowb.,

414. aviculoides Rv.,

415. emarginata Sowb.,

416. gradata Brod.,

417. grandis Brod.,

418. mutabilis Sowb.,

419. pholadiformis Ad.,

420. Reeveana Orb.,

421. reversa Sowb.

422. similis Ad.,

423. solida Sowb.,

424. Tabogensis Ad,

425. tuberculosa Sowb.,

426. sp. indet,

\section{Family Cardiidae.}

CARDITA.

427. C. affinis Sowb.,

428. laticostata Sowb.,

429. radiata Sowb.,

430. graniferum Brod.,
431. C. obovale Brod, 267

432. planicostatum Sowb., 267

433. procerum Sowb., 267

434. senticosum Sowb., 268

\section{Family Veneridae.}

VENUS.

435. V. amathusia Phil., $\quad 269$

436. discors Sowb., 269

437. gnidia Brod., 269

438. multicostata Sowb., 270

439. pectunculoides Val., 270

440. subrugosa Sowb. 271

441. sp. indet. $a, \quad 271$

442. " b. b. 271

CYTHEREA.

443. C. affinis Sowb.,

444. aurantiaca Hanley, 272

445. consanguinea Ad., 272

446. radiata Sowb., 273

447. squalida Sowb., 273

ARTEMIS.

448. A. Dunkeri Phil, 274

449. saccata Gould,

GOULDIA.

450. G. Pacifica Ad.,

CYRENA.

451. C. maritima Ad.,

275

\section{Family Tellenida.}

LUCINA.

452. L. tellinoides $R \mathrm{r}$.

CAPSA.

453. C. altior Sowb.,

DONAX.

454. D. assimilis Hanley,

455. gracilis Hanley,

456. navicula Hanley,

278

457. rostratus Ad.,

278

458. T. aurora Hanley, 
No.

459. T. cognnta Ad.,

460. Columbiensis Hanley,

461. concinna Ad,

462. crystallina Chem.,

463. Cumingii Hanley,

464. Dombei Hanley,

465. felix Hanley,

466. laceridens Hanley,

467. prora Hanley,

468. puella Ad.,

469. rubescens Hanley,

470. siliqua $\mathrm{Ad}$,

471. simulans Ad,

472. sincera Hanley,

473. vicina Ad.,

474. sp. indet. $a$,

475. " $\quad b$,

476. " $c$

\section{Family Petricolidae.}

PETRICOLA.

477. P. cognata Ad.,

SAXICAVA.

478. S. tenuis (?) Sowb.,

\section{Family Mactridae.}

CUMINGLA.

479. C. coaretata Sowb,

48a. trigenularis Sowb,

481. sp. indet. $a$,

482. " $b$,

483. " " $c_{3}$

484. " $\quad d$,

AMPHUDESMA.

485. A. bicolor Ad,

486. ellipticum Sowb,

487. proximum Ad.,

488. pulchrum Sowb,

489. striosum Ad.,

490. tortuosum Ad.,

491. ventricosum Ad.,

CRASSATELLA.

492. C. gibbosa Sowb.,

MULINIA.

493. M. donaciformis Hanley,

494. ventricosa Gould,
Page 279

280

280

281

281

282

282

282

283

283

283

284

284

285

285

286

286

286

286

287

1

$\begin{array}{ll}\text { 505. ovulata Sowb, } & 298 \\ 506 . & \text { rubra Ad., }\end{array}$

507. tenuis Sowb, 299

508. sp. indet. $a, 300$

509. " $b, \quad 300$

287

288

288

288

288

288

288

289

289

290

291

291

292

292

293

293

No.

LUTRARTA.

495. L. elegans Sowb.,

Page

mactra.

496. M. velata Phil.,

294

294

\section{Family Corbulidae.}

ANATINA.

497. A, alta Ad,

294

295

295

296

296

297

297

298

9

\section{Family Solenidae.} SOLECURTUS.

510. S. affinis Ad,

300

SOLEN,

511. S. rudis Ad.,

300

\section{Family Pholadida.}

PHOLAS.

512. P. crucigera Sowb., 301

513. tubifera Sowb., 302

514. xylophaga Val. (non al.), 302

515. sp. indet. $a, \quad 302$

516. " $b$, 302

\section{Class Brachiopoda.}

orbicULA.

517. O. Cumingii Brod. 


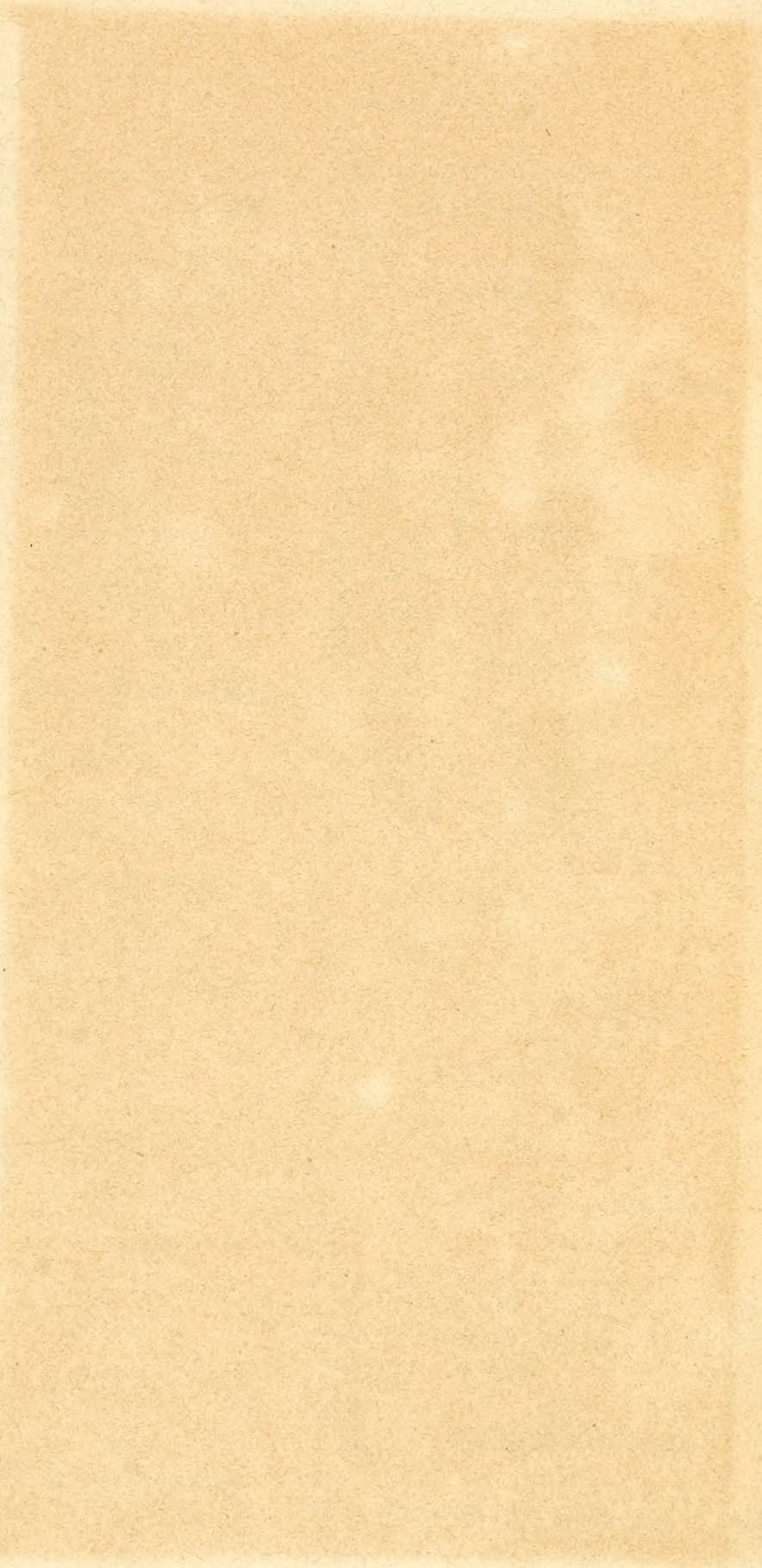



